\title{
Analytical and numerical studies of Bose-Fermi mixtures in a one-dimensional harmonic trap
}

\author{
A. S. Dehkharghani, F. F. Bellotti, and N. T. Zinner \\ Department of Physics and Astronomy, Aarhus University, DK-8000 Aarhus C, Denmark
}

\begin{abstract}
In this paper we study a mixed system of bosons and fermions with up to six particles in total. All particles are assumed to have the same mass. The two-body interactions are repulsive and are assumed to have equal strength in both the Bose-Bose and the Fermi-Boson channels. The particles are confined externally by a harmonic oscillator one-body potential. For the case of four particles, two identical fermions and two identical bosons, we focus on the strongly interacting regime and analyze the system using both an analytical approach and DMRG calculations using a discrete version of the underlying continuum Hamiltonian. This provides us with insight into both the ground state and the manifold of excited states that are almost degenerate for large interaction strength. Our results show great variation in the density profiles for bosons and fermions in different states for strongly interacting mixtures. By moving to slightly larger systems, we find that the ground state of balanced mixtures of four to six particles tends to separate bosons and fermions for strong (repulsive) interactions. On the other hand, in imbalanced Bose-Fermi mixtures we find pronounced odd-even effects in systems of five particles. These few-body results suggest that question of phase separation in one-dimensional confined mixtures are very sensitive to system composition, both for the ground state and the excited states.
\end{abstract}

\section{INTRODUCTION}

One-dimensional quantum systems have been some of the most widely used examples in the theoretical physics literature [1 [5], mostly due to their simplicity in contrast to higher dimensions. Moreover, recent experimental realizations with cold atoms in one dimension (1D) [6 17] have made it possible to test new and old theories for such systems. In recent years, experimental manipulations have evolved so rapidly and in many new ways that one can now build a Fermi sea one atom at a time in low-dimensions [18 23] and therefore be able to study and understand the transition between few- and manybody systems [24, 25]. Other existing predictions such as some from the Bethe ansatz [26 29] and that of the Tonks-Girardeau gas 30, 31 have also been studied and tested successfully [11, 13, 14, 32, 33]. Even mixing different kinds of particles and species with tunable interaction between the atoms is now feasible 22, 34] and therefore opening up for developing and testing new kinds of theories.

Despite being simpler than higher dimensions, 1D systems are sometimes more interesting and exotic [19]. This is mainly due to the 1D nature that prohibits any exchange of particles without their wave function overlapping with each other. At the same time, this allows one to build a chain of atoms with one or several internal components and start manipulating them by changing the surroundings [19, 35] or tuning the interactions between the particles through Feshbach Resonances [36, 37]. In the long term, this can be used for transport of quantum information [38] and such knowledge may be advantageous in a variety of technological applications such as nano-tubes and nano-wires [39].

At the theoretical level 1D systems have been intensively studied both analytically and numerically in the recent years 40 66. Numerically, the DMRG method has been one of the most successful methods. It was firstly developed for discrete Hamiltonians [67 70$]$ and then pushed to the limit of continuous systems even for strongly interacting particles 71,72 . Analytically, new and different kinds of methods have been developed and are in use as well $73-76]$. However, different kind of methods for all regimes have both advantages and disadvantages, and they must be used with care [77].

Here we study both numerical DMRG methods and analytical exact solutions of Bose-Fermi mixtures in a one-dimensional harmonic trap in the limit of strong interaction, and we compare how well the DMRG method captures the physics of the system. In addition, we compare our results with other recent studies of such systems 71, 72, 78 82]. In particular, the study of Deuretzbacher et al. 78] have recently considered a spin-chain model approach to Bose-Fermi mixtures, which is similar to the analytical approach to the strongly interacting limit that we use below. Our analytical results are in agreement with the work in [78].

The structure of the paper is as follows. In Section II we present our systems and the methods. Boson-fermion mixtures are discussed in Section $\amalg$ in connection with fermionization of such systems in the strongly interacting limit. Concluding remarks are given in Section IV]

\section{SYSTEM AND METHODS}

Our system consists of a mixture of bosons and fermions with total number of particles $N=N_{b}+N_{f}$, where $N_{b}$ denotes the number of identical bosons and $N_{f}$ is the number of identical fermions. All the particles have the same mass $m$ and are confined with the same trap frequency $\omega$ in a one-dimensional harmonic trap, $V(x)=m \omega^{2} x^{2} / 2$. The two-body interaction is short-range and we model it by the zero-range model as 
a Dirac $\delta$-function. Furthermore, we assume that the interactions are purely repulsive throughout this paper. The full Hamiltonian can therefore be written as

$$
\mathcal{H}_{c}=\sum_{i=1}^{N}\left(-\frac{\hbar^{2}}{2 m} \frac{\partial^{2}}{\partial x_{i}^{2}}+V\left(x_{i}\right)\right)+\sum_{i<j}^{N} U_{i j}\left(x_{i}-x_{j}\right),
$$

with the interaction matrix $U_{i j}\left(x_{i}-x_{j}\right)=g_{i j} \delta\left(x_{i}-x_{j}\right)$ and $g_{i j}>0$. The interaction strength is assumed to be the same $g_{i j}=g$ if particle $i$ and particle $j$ are either from different species or they are identical bosons with selfinteraction. However, due to the Pauli exclusion principle we may set $g_{i j}=0$ when $i$ and $j$ are fermions as identical fermions will not feel any effect of a zero-range potential.

In order to study the system using DMRG in the usual discrete form, we need to specify a lattice model for the Hamiltonian above. Here we take discrete lattice Hamiltonian of the form

$$
\begin{gathered}
\mathcal{H}_{d}=-t \sum_{j=1}^{N-1}\left(b_{j}^{\dagger} b_{j+1}+b_{j+1}^{\dagger} b_{j}\right)-t \sum_{j=1}^{N-1}\left(f_{j}^{\dagger} f_{j+1}+f_{j+1}^{\dagger} f_{j}\right)+U_{b f} \sum_{j=1}^{N} n_{b, j} n_{f, j} \\
+\frac{U_{b b}}{2} \sum_{j=1}^{N} n_{b, j}\left(n_{b, j}-1\right)+V_{h} \sum_{j=1}^{N}(j-L / 2)^{2}\left(n_{b, j}+n_{f, j}\right)
\end{gathered}
$$

where $b_{j}$ and $f_{j}$ are the bosonic and fermionic field operators, respectively, acting on a site $j$ and with the corresponding density operators $n_{b, j}=b_{j}^{\dagger} b_{j}$ and $n_{f, j}=f_{j}^{\dagger} f_{j}$. The tunneling constant, $t$, is the equivalent of the kinetic term in the continuous case, while $U_{b f}$ and $U_{b b}$ are the on-site interactions. The strengths of the on-site interactions are correspondingly assumed to be the same, $U_{b f}=U_{b b}=U$, and the strength of the harmonic potential is called $V_{h}$. In the low-density limit where the number of particles is much less than the number of discrete lattice sites, this model should reproduce the physics of the continuous system.

In the limit of very strong interaction strengths an ex- act analytical wave function has been derived [74, 75, 83. for the continuous Hamiltonian in Eq. (1). This method makes use of the fact that up to linear order in $1 / \mathrm{g}$ one can obtain the exact slope of the eigenstates and use this fact to derive an analytical solution for each eigenstate. The method is applicable for any arbitrary confining geometries and in a particular case with a harmonic trap. The case of four particles with two-component fermions has been treated and derived in details recently [77]. The main result here is that the wave function of the twocomponent mixture system with $N=N_{b}+N_{f}$ particles can be written as a non-trivial combination of the antisymmetric product of the first $N$ eigenstates to the non-interacting part of Eq. (1)

$$
\psi\left(x_{1}, \ldots, x_{N}\right)= \begin{cases}a_{1} \Psi_{A} & \text { for } x_{b_{1}}<\ldots<x_{b_{N_{b}}}<x_{f_{1}}<\ldots<x_{f_{N_{f}}}(b \ldots b f \ldots f) \\ a_{2} \Psi_{A} & \text { for } x_{b_{1}}<\ldots<x_{f_{1}}<x_{b_{N_{b}}}<\ldots<x_{f_{N_{f}}}(b \ldots f b \ldots f) \\ \vdots & \vdots \\ a_{M} \Psi_{A} & \text { for } x_{f_{N_{f}}}<\ldots<x_{f_{1}}<x_{b_{N_{b}}}<\ldots<x_{b_{1}}(f \ldots f b \ldots b)\end{cases}
$$

where $M=N ! /\left(N_{b} ! N_{f} !\right)$ denotes the number of degeneracy at $1 / g \rightarrow 0$ and $x_{n}$ is the coordinate of the $n^{\text {th }}$ particle. The coefficients, $\left\{a_{1}, a_{2}, \ldots, a_{M}\right\}$ are then found by considering the slope of the energy as one approaches $1 / g \rightarrow 0$.

To solve the lattice model we will use DMRG as stated above. In recent years, many libraries that implement the DMRG method [70, 84, 85] have been developed to solve discretized lattice Hamiltonians. In this article we make use of two independently developed open-source codes, one from L. D. Carr and his group [70, 84] and the other from the iTensor project [85]. We find consistency of the results from both codes.

A mapping between the discrete and continuous Hamiltonian has also been investigated and described recently 77. . One of the important mappings here is the connection between the interaction strength $g$ and the on-site interaction $U$, which is found to be the following

$$
U=0.10291 \cdot g
$$

The above relation is found by investigating the relation between the energy calculated from the discrete Hamiltonian Eq. (2), labeled $E_{d}$, and the one from the continuous 
Hamiltonian Eq. (10), labeled $E_{c}$, which is given by

$$
\frac{E_{c}}{\hbar \omega}-N \frac{1}{2}=\frac{E_{d}}{\hbar \omega_{d}}-N \frac{E_{d_{1 \mathrm{p}}}}{\hbar \omega_{d}} .
$$

Here $E_{d_{1 \mathrm{p}}}$ is the energy one obtains when using the discrete model to solve the one particle system in a harmonic trap and $\hbar \omega_{d}$ is the energy difference between the ground and 1st excited states. $\hbar \omega_{d}$ is important to calculate in order to know the units of the discrete model. $E_{c}$ is the energy obtained by the continuous model and $N$ is the total number of particles. The second term on both sides subtracts the non-interacting ground state energy in the same units as the first term.

With this mapping one can compare the DMRG results with the exact solution in the strongly interacting regime. Since the exact solution is only valid in the $1 / g \rightarrow 0$ limit, one can use other methods, such as variational 73 . or exact diagonalization [45, 60] to compare the results for intermediate values of $g$. However, in this work we only focus on the DMRG results for the strong interacting regime in a mixture of bosons and fermions and how these results compare to the ones obtained by the exact methods. In addition, we are also interested in how the particles mix with the opposite species.

\section{MIXED SPECIES SYSTEMS}

In the following we study mixtures of identical bosons (denoted by $b$ ) and identical fermions (for concreteness we take them to have spin up) [61, 62]. We first consider the case study of a four-body mixture of equal numbers of bosons and fermions. Then we proceed to study systems with five and six particles in order to generalize our findings and investigate what we may inferred about larger systems from our few-body approach.

\section{A. Two bosons and two fermions}

First we take $N_{\uparrow}=N_{b}=2$. All particles are assumed to interact with a zero-range potential of strength $g$. Due to the Pauli principle the fermionic particles are antisymmetric under inter-particle exchange and are therefore not affected by this zero-range interaction. The degenerate manifold of states for strong interaction will have $M=4 ! /(2 ! 2 !)=6$ states. The mixture system is of particular interest since there have been speculations that the system will fermionize in a trivial way so that all particles will behave as essentially identical fermions 71 . In Ref. 71] this conclusion was supported by both analytical and DMRG calculations. However, one may use the techniques of Ref. [74] and detailed calculations of Ref. [77] for the two-species system to show that the ground state does not fermionize in this manner.

In figure 1we show the six states for the two bosons and two fermions mixture in the strongly interacting regime.
In the center of the figure we display the slopes of the energy close to $1 / g \rightarrow 0$, while along the edge of the figure we show the density of profiles of the six states enumerated in accordance with the slopes. The fermion density is shown as a solid (black) line while the bosons are show by a dashed (red) line. The first observation to make is that the ground state (denoted state 0) has a very specific manner in which it fermionizes; the two bosons are seen to be pushed to the center of the trap while the two fermions are left to occupy the edges. This may be intuitively understood from a tendency for the fermions to want to avoid each other, but we caution that since all the particles have strong two-body repulsive interactions, it is a subtle issue. The bosons could also be said to want to avoid each other as much as possible. In the competition of these effects, we see that the Pauli principle for the fermions seem to imply a preference for bosons in the middle and fermions out to the sides.

A particularly peculiar second observation, is that there is an excited state (denoted state 3 ), where the trend of the ground state is exactly reversed, i.e. bosons go the sides while fermions go to the middle. Keep in mind that in the strict limit where $1 / g=0$, all states are energetically degenerate, again pointing out the very subtle dynamics that determines the spatial configurations. A similar story is seen to happen when comparing states 2 and 5 in figure 1, exact opposite trends of spatial distributions of bosons and fermions are witnessed. The final two states denoted 1 and 4 are seen to have completely 'democratic' fermionization, i.e. the density profiles look exactly like a system of four identical fermions. Note that even though these two states look identical, their fourbody wave functions are distinct and they are orthogonal states due to different signs of different configurations in the wave functions. In a density plot this is not visible as it depends on the absolute value squared of the wave functions. In comparison to the ground state discussed in Ref. 71] which has a density profile like states 1 and 4 in our figure 1 and which was supported by DMRG evidence in [71], we speculate that this state may have been an excited state that the DMRG could not resolve due to the (quasi)-degenerate nature of the spectrum for very strong interactions which can make DMRG calculations unreliable [77].

The example in figure 1 highlights how the spatial structure of the system is very different for different states in the strongly interacting regime. It indicates that if one can find a way to selectively populate the excited states of the system, one would have access to preparation of few-body systems with distinct quantum spatial structure, and in turn to tailoring of quantum magnetism of mixed systems in small systems.

In order to compare the analytical results of figure 1 , we perform DMRG calculations and show the density profiles in figure 2. Indeed, one can get to the strongly interacting regime and reproduce the analytical profiles, but this must be done with care. Figure 2 shows the evolution of the density profiles as we go from the non- 


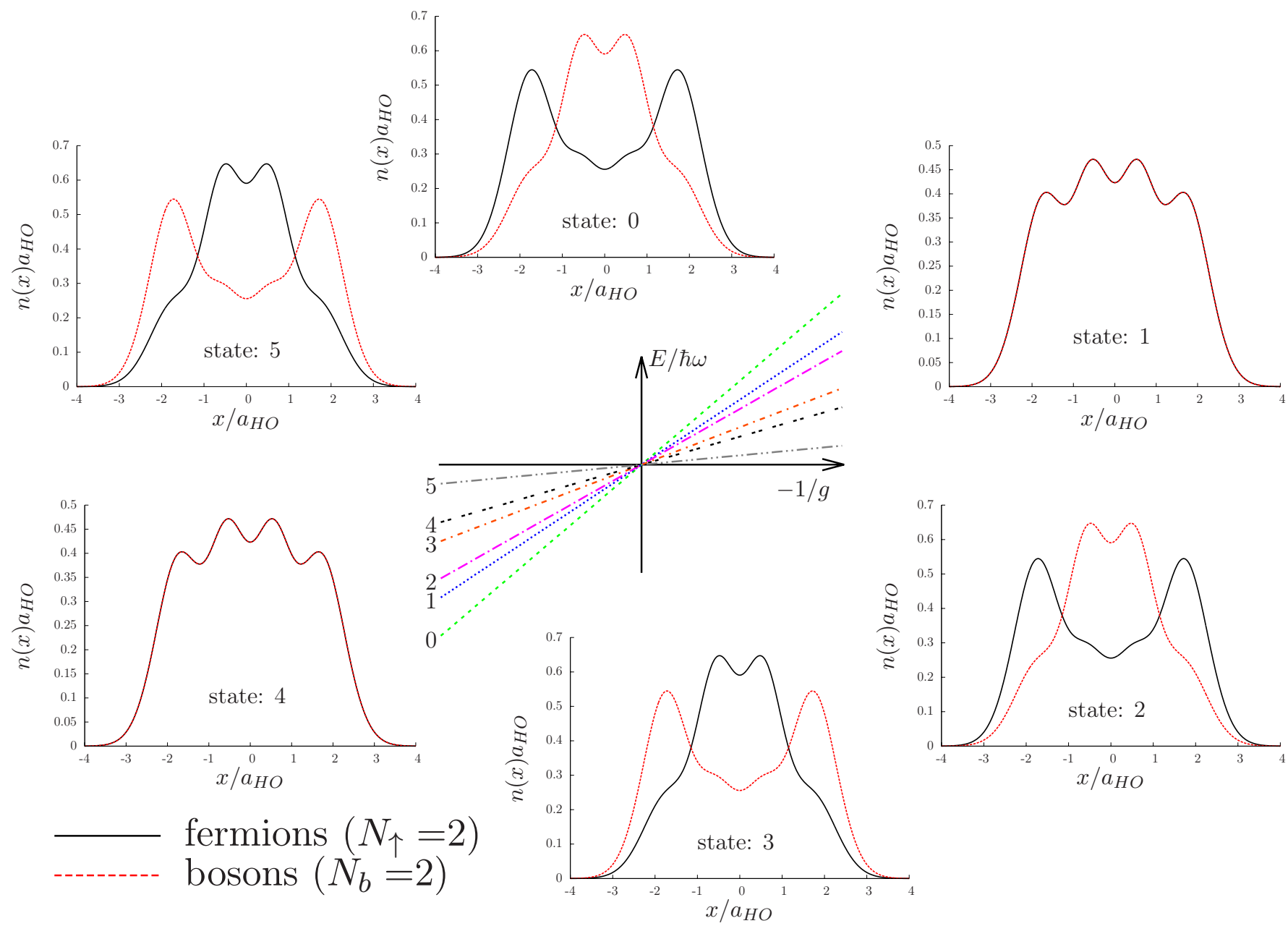

FIG. 1. Energy slopes for the 6 degenerated states at $1 / g=0$ and density profiles for the six states for a mixture of 2 fermions and 2 bosons. Results obtained with the wave function Eq. 3 following the procedure from 74 ].

interacting, $g=U=0$, to the strongly interaction regime where $g \sim 100$ and in turn $U \sim 10$. All the points in the figure have been calculated with DMRG while the solid lines in figure 2(d) are from the analytical calculations of the density profiles as discussed above. We do see a bit of deviation from the analytical profiles but not alarmingly so. This does show clearly the need for analytical insights in this regime.

In order to further analyze the four-body mixture, we focus on the spatial configurations in more detail. The six states that become degenerate at $1 / g=0$ are superpositions of the $M=6$ independent ways to order the two bosons and two fermions on a line. The amplitude of a given configuration of the four bodies is highly nontrivial and the weights must be determined by solving for the coefficients $a_{k}$ in Eq. (3). This can be done in the manner outlined in 74].

To illustrate the spatial configurations it is advantageous to consider the pair correlation function between the bosons and the fermions which contains information on their relative positions in the harmonic trapping po- tential. The pair correlation is given by

$\mathrm{P}(x, y)=\int d x_{1} \ldots d x_{N} \delta\left(x-x_{1}\right) \delta\left(y-x_{N}\right)\left|\psi\left(x_{1}, \ldots, x_{N}\right)\right|^{2}$.

In the present case we have $N=4$ and since there are two different kinds of particles, the particles that correspond to coordinates $x_{1}$ and $x_{4}$ will be a boson and a fermion, respectively, as can be verified by considering the analytical form of the wave function in Eq. (3). We can therefore also refer to this pair correlation as the boson-fermion correlation function. Some examples of spatial configurations are shown in figure $3(\mathrm{a}),(\mathrm{b})$ and (c). The panels show spatial configurations $\uparrow \uparrow b b$ (associated to the amplitude $a_{1}$ in Eq. (3) ) in panel (a), $\uparrow b \uparrow b$ (coefficient $a_{2}$ ) in panel (b) and $\uparrow b b \uparrow$ (coefficient $a_{3}$ ) in panel (c). In panel (a) we see the pair correlation focused in the upper left-hand corner where boson-boson and fermion-fermion distances are small, while the boson-fermion distance has two maximum that are rather close still, and similarly for panels (b) and (c). Notice that the configurations $b \uparrow \uparrow b$ (coefficient $a_{4}$ ), $b \uparrow b \uparrow\left(\right.$ coefficient $\left.a_{5}\right)$ and $b b \uparrow \uparrow\left(a_{6}\right)$ are 

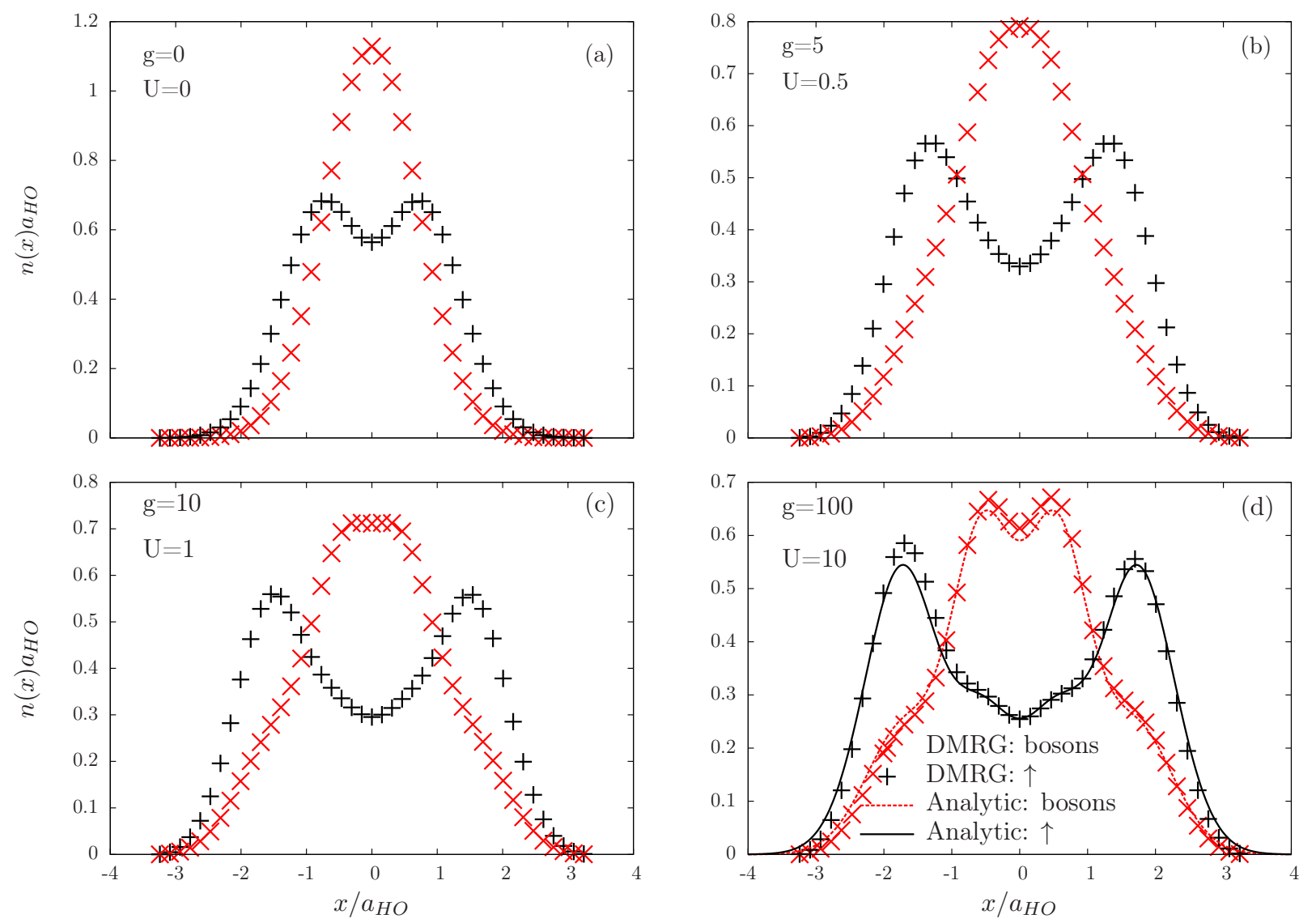

FIG. 2. DMRG results for the evolution of the ground state density profile as function of the interaction parameter $g$ ( $U=$ $0.10291 \mathrm{~g}$ ). Lines on the last panel are the exact solution in the strongly interacting limit Eq. (3). The parameters of the DMRG calculations for the Hamiltonian in Eq. (2) are $V_{h} / t=7 \cdot 10^{-6}, t=1, L=128$, and $U_{b f}=U_{b b}=U$.

not shown but may be very easily obtained by switching bosons and fermions which corresponds to a reflection in the $x_{b}=x_{f}$ plane in panel (a), (b) and (c) in figure 3

Let us first consider the analytical wave functions in the strongly interacting limit. The amplitudes of the different spatial configurations as given in Eq. (3) are

$$
\begin{aligned}
& \left(a_{1}, a_{2}, a_{3}, a_{4}, a_{5}, a_{6}\right)= \\
& (0.222,0.448,0.669,0.226,0.448,0.222),
\end{aligned}
$$

for the ground state and

$$
\left(a_{1}, a_{2}, a_{3}, a_{4}, a_{5}, a_{6}\right)=(0.5,-0.5,0.0,0.0,0.5,-0.5)
$$

for the first excited state. The combination of the six spatial configurations properly weighted by their coefficients $a_{k}$ gives the pair correlation function shown in Figure 3(d) for the ground state and in Figure 3(g) for the first excited state (the respective densities can be seen in Figure 1). For the ground state, we see that the dominant configuration corresponds to amplitude $a_{3}$, i.e to the two bosons sitting in the center and the fermions on the edge of the trap as seen in the densities of figure 1 However, there are considerable contributions from all the other configurations as well. In the pair correlation function this is reflected since panel (d) for the ground state has its dominant non-zero contributions in the same places as that seen for the configuration corresponding to coefficient $a_{3}$ as seen in panel (c). The difference comes from a large admixture of the configuration in panel (b) and a smaller contribution from that in panel (a). The first excited state is much more 'clean' in the sense that it only has configurations corresponding to coefficients $a_{1}$ and $a_{2}$, and with opposite signs. Panel (g) in figure 3 therefore corresponds to a superposition of the results shown in panel (a) and (b).

We note that these results also show that there is no simple relation among the coefficients of the configurations in general. For instance, if we consider a system of three equal mass particles where two are identical fermions, an allowed state for $1 / g=0$ is

$$
\begin{aligned}
& \psi_{(2+1)}\left(x_{1}, x_{2}, x_{3}\right)= \\
& \left(x_{1}-x_{2}\right) \cdot\left|x_{2}-x_{3}\right| \cdot\left|x_{1}-x_{3}\right| \cdot \exp \left(-x_{1}^{2}-x_{2}^{2}-x_{3}^{2}\right),
\end{aligned}
$$

where $x_{1}$ and $x_{2}$ are the coordinates of $N_{\uparrow}$ and $x_{3}$ the coordinate of $N_{\downarrow}$. This wave function is inspired by a Tonks-Girardeau state which can be written as a Vandermonde determinant 61]. But this is not a ground state and does not yield a state that is adiabatically con- 

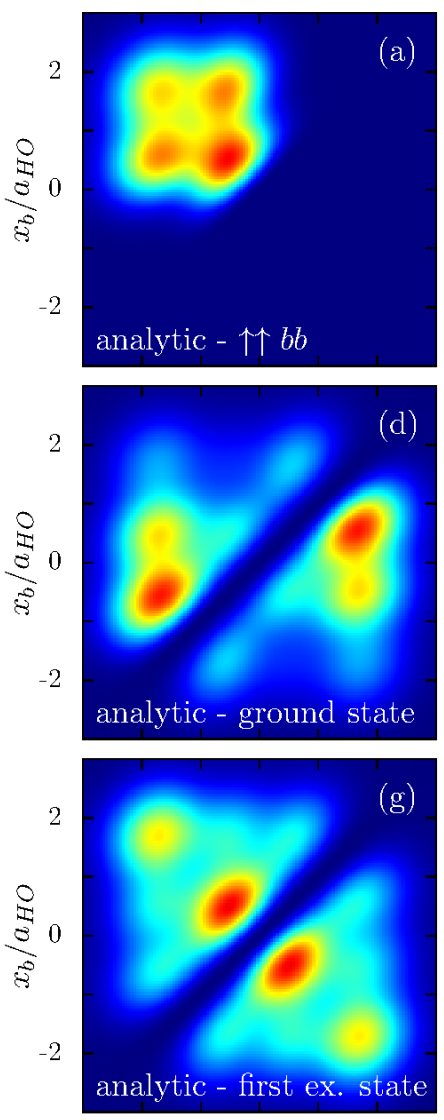

$-2$

2
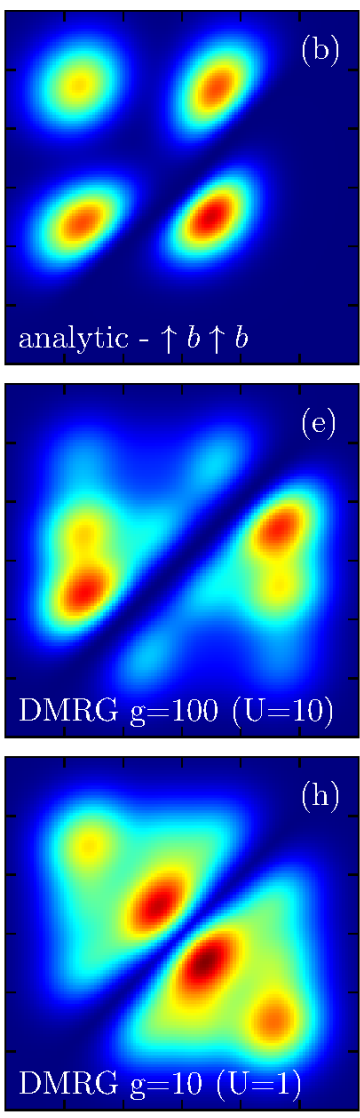

$-2$

2
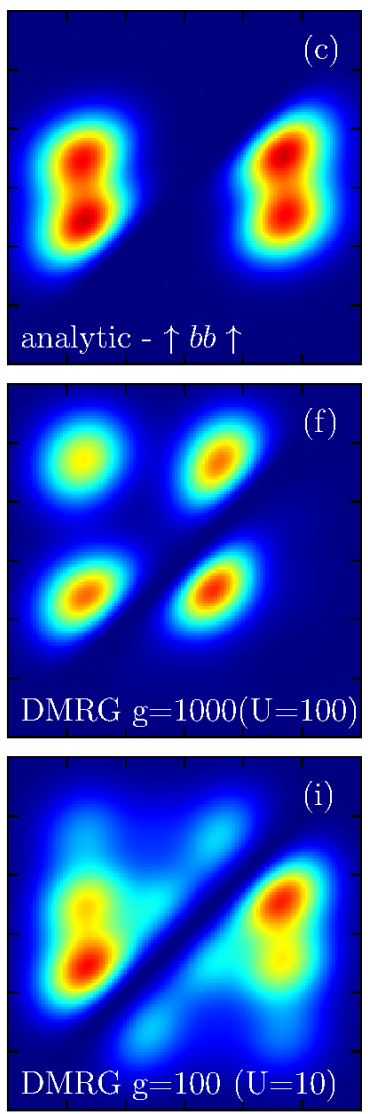

$x_{\uparrow} / a_{H O}$

0
$\uparrow / a_{H O}$

$x_{\uparrow} / a_{H}$

FIG. 3. Pair correlation functions of a $N_{\uparrow}=N_{b}=2$ system. Panels (a) to (c) show the analytical results respectively for the spatial configurations $\uparrow \uparrow b b, \uparrow b \uparrow b$ and $\uparrow b b \uparrow$. Panels (d) and (g) give analytical results for ground and first excited states. DMRG results for the ground state are presented in panels (e) and (f) for the first excited state in panels (h) and (i). The parameters used for the DMRG calculations are as given in the caption of figure 2

nected to other states in the spectrum for large but finite interaction strength [74]. The relation of the exact solutions and the wave functions obtained from these Vandermonde inspired wave functions has been discussed in detail in Ref. [63]. Note that if we are discussing two identical bosons and a third particle, then making the substitution $\left(x_{1}-x_{2}\right) \rightarrow\left|x_{1}-x_{2}\right|$ in the wave function above will in fact give us a valid ground state as long as all two-body interactions are equal and large as it becomes the Girardeau wave function [31] which is the ground state also for two-component bosons with equal interactions between all pairs [42].

In closing this section, we compare the pair correlation functions obtained from the analytical methods to the DMRG results. For the ground state, these are shown in panel (e) and (f) in figure 3. Here we see that there is quite a good resemblance of the DMRG pair correlation results for $U=10$ and that for the analytical results in panel (d). This can be immediately contrasted with the results from DMRG for $U=100$ as shown in panel (f) which clearly looks nothing like the ground state. Curi- ously, it looks instead more or less perfectly like the results shown in panel (b) in figure 3. This would lead us to conclude that the DMRG predicts that the wave function is that of the single spatial configuration with structure $\uparrow b \uparrow b$ which is of course not the case. Changing slightly the parameters and initialization of the DMRG routine may prompts the system to get stuck in some other spatial configuration as all of these become degenerate for extremely large interactions such as $U=100$ corresponding to $g \sim 1000$. For the first excited state the DMRG results are shown in panel (h) of figure 3 and this looks very similar to the analytical results in panel $(\mathrm{g})$. However, notice here that we have a quite small $U=1$ and thus $g \sim 10$. For larger $U$ we again get stuck in the wrong kind of state as shown in panel (i), this time in something that looks very similar to the ground state wave function as given in panel (d). We find that for higher excited states one should generally expect that the strongly interacting limit sets in at smaller values of $U$ as compared to the ground state, see also [77. 


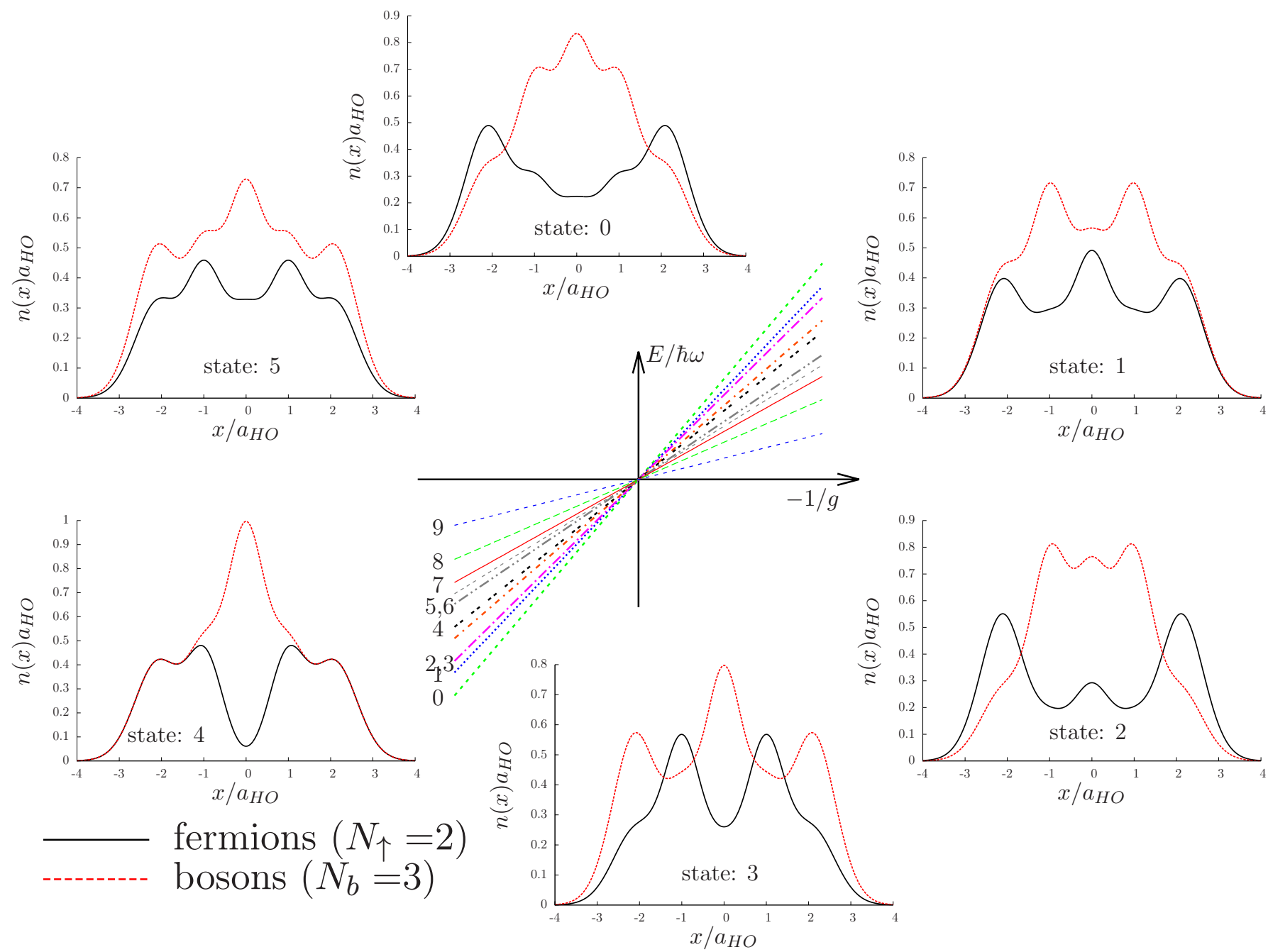

FIG. 4. Energy slopes for the 10 degenerated states at $1 / g=0$ and density profiles for the six lowest lying states for a mixture of 2 fermions and 3 bosons. Results obtained with the wave function Eq. 3 following the procedure from [74].

\section{B. Larger systems}

Next, we analyze mixtures with a higher number of particles in order to look for some trends in larger systems. We first analyze mixtures with $N_{\uparrow}=2$ and $N_{b}=3$, which have $M=5 ! /(2 ! 3 !)=10$ degenerated states at $1 / g=0$. This is an odd-even mixture similar to the case highlighted in Ref. [78], although here for a smaller total number of particles. The results for the energies to linear order in $1 / g$ near $1 / g=0$ are shown in figure 4 as obtained from Eq. (3). Along the edge of the figure we show the density profiles of a subset of the states.

The density profiles for the first six states in figure 4 now become more elaborated and some states have the bosons and fermions tending to intercalate (sit in between each other). This is very different from the case with $N_{\uparrow}=N_{b}=2$ above, where there are two kinds of configurations, either species in the center and the other on the side, or the 'democratic' fermionization corresponding to a density akin to four identical fermions. This latter kind of order is not seen in any of the ten states for the five-body case. A state that is somewhat like the democratic choice is that denoted state 4 in figure 4 which corresponds roughly to a boson occupying the center alone, and then on the wings we have equal amounts of bosons and fermions. Note also that we do not find state where the fermions dominate the occupancy in the center region. The ground state and state 2 are seen to have separated densities (akin to ferromagnetism), while states 1 and 3 have alternating boson-fermion structure. In particular, state 3 seems to carry a strongly antiferromagnetic tendency. The ground state profile seen here is consistent with that found in the odd-even $N=17$ case studied in Ref. [78].

The next case we consider is one in which we still have five particles but this time with $N_{\uparrow}=3$ and $N_{b}=2$. Again, there are $M=10$ states that become degenerate in energy at $1 / g=0$. The results for this system are shown in figure 5. The linear slopes of the energy around $1 / g=0$ are only slightly different from the other fivebody case above. However, the ground state profile is now very different as it shows clear intercalation between 


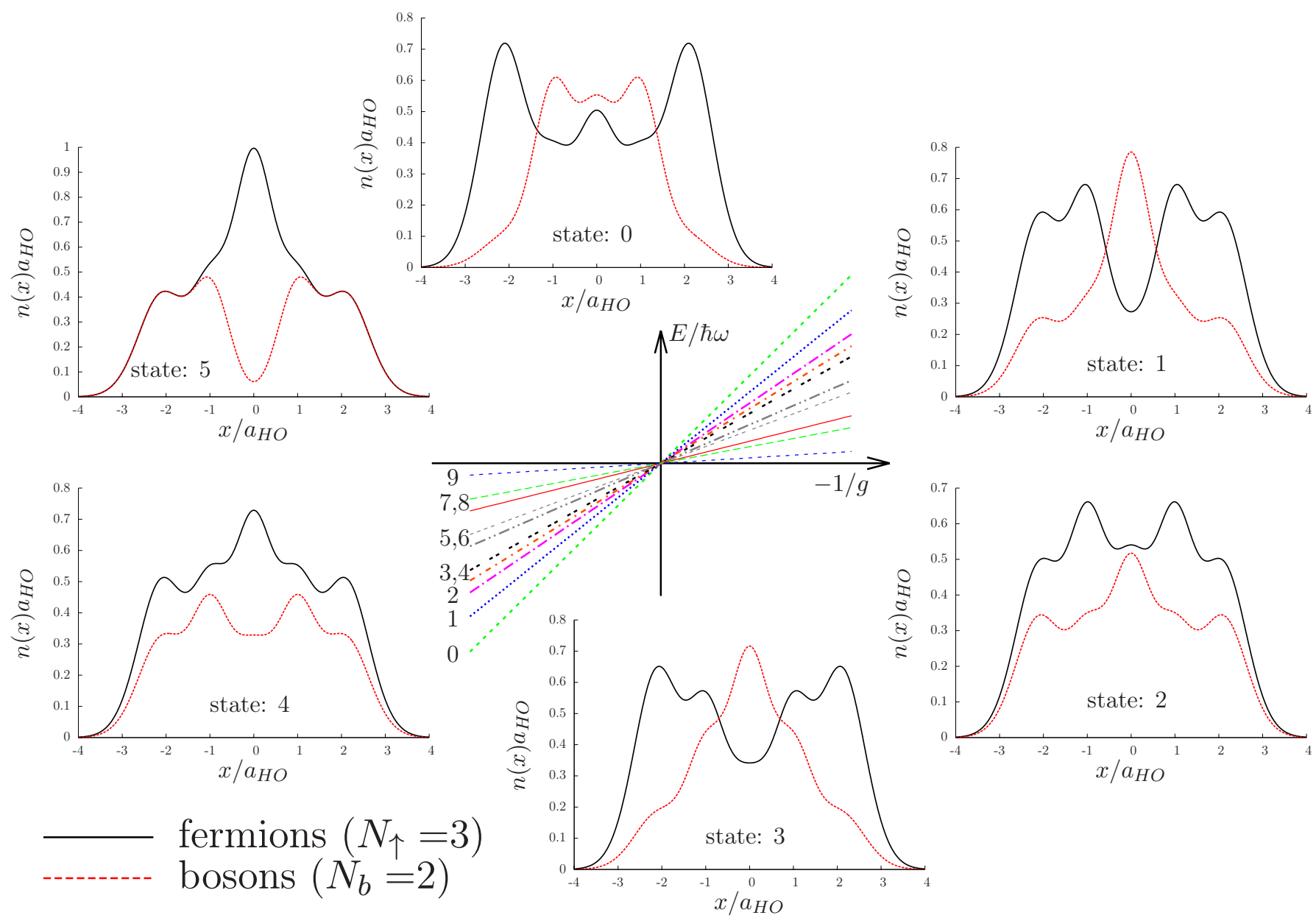

FIG. 5. Energy slopes for the 10 degenerated states at $1 / g=0$ and density profiles for the six lowest lying states for a mixture of 3 fermions and 2 bosons. Results obtained with the wave function Eq. 3 following the procedure from [74].

the bosons and fermions, while with a majority of bosons above we saw the bosons push the fermions out of the center of the harmonic trap. Interestingly, we see that the states denoted 2 and 4 in figure 5 are very similar in their structure if one interchanges the bosons with fermions. However, the other profiles are completely different. As before, systems composed of $N_{\uparrow}=3$ and $N_{b}=2$ do not possess any democratically fermionized state resembling that of five identical fermions in their density profiles.

The discussion of the balanced four-body system in the previous section and the two ways to imbalance the system in the five-body case lead is to conclude that even in the case of these relatively modest size systems one may have very pronounced odd-even effects in BoseFermi mixtures.

The last system we consider is a balanced setup of fermions and bosons with $N_{\uparrow}=N_{b}=3$. In this case we have $M=20$ degenerate states at $1 / g=0$. The slope of the energy around $1 / g=0$ for all these states are shown in figure 6 along with the density profiles for the six lowest lying states. In the ground state, bosons are again pushed to the center of the trap and two fermions are clearly sitting at the edge of the trap. However, a third fermion seems to be sticking around between the bosons close to the origin. This time we do see the presence of a democratically fermionized state which is the sixth excited state for which the density is also plotted in figure 6 . We also see some rather peculiar mixed density profiles in the manifold of excited states. Consider for instance the state denoted by 2 in figure 6 which seems to have all the bosons centered around the middle, but at the same time similar peaks in the fermionic density. This could indicate that a single fermion is sitting at the edge while the other two fermions are 'democratically' mixing with the bosons in the center. In comparison to the four- and five-body cases we do see some similar trends, and again we see strong odd-even effects when going from five to six particles.

\section{CONCLUSION}

We studied Bose-Fermi mixtures of four, five and six particles in both balanced and imbalanced systems. The 


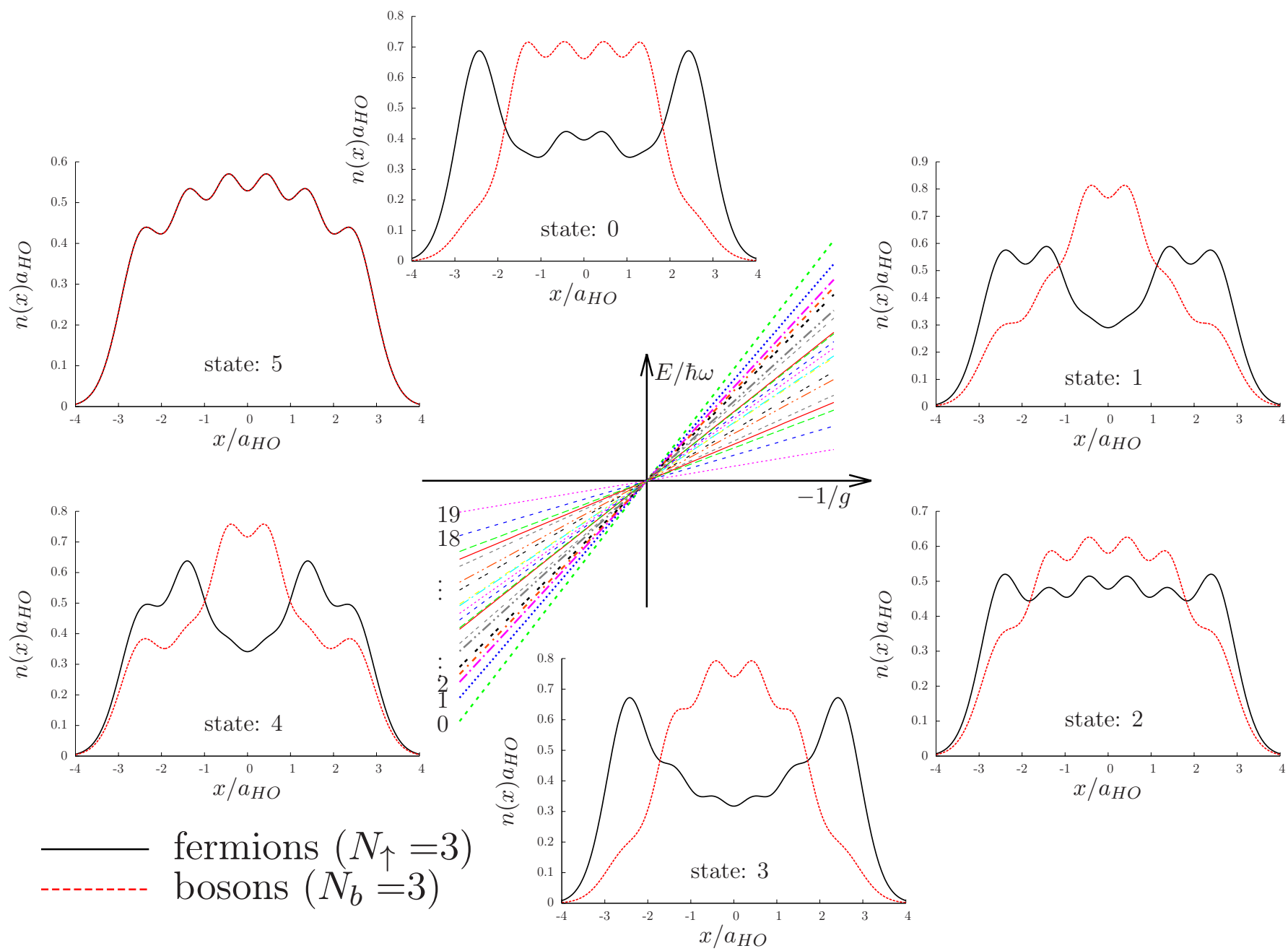

FIG. 6. Energy slopes for the 20 degenerated states at $1 / g=0$ and density profiles for the six lowest lying states for a mixture of 3 fermions and 3 bosons. Results obtained with the wave function Eq. 3 following the procedure from [74].

ground state of four and six particles systems with equal number of bosons and fermions turns out to try to separate the two kinds, bosons in the middle of the trap and fermions out on the wings. For imbalanced systems with five particles, we find that the particles would rather like to blend together, i.e. they seem to be miscible in imbalanced systems. This suggests that phase separation of Bose-Fermi mixtures is an extremely delicate question in the few-body case [66]. In fact, even the density profile of the ground state depends sensitively on whether it is the bosons or fermions that are present in an odd number. Previous studies in large homogenous systems 79 81 also suggest that the question of whether the bosons and fermions mix or not is very delicate and depends on the theoretical approach. The study in Ref. 81] does find good agreement with our results in the few-body limit by using a local density approximation for the harmonic trap. A study using density-functional theory in a harmonic trap 82] seem to agree with our few-body finding of separation for balanced systems, but also finds evidence for separation (fermions pushed to the side of the trap) in imbalanced systems where we find a larger tendency for mixing. In particular, for the case with more fermions than bosons, we find that a fermion can penetrate the bosonic density, which seems not to be the case in Ref. 82]. This may become less pronounced for larger particle numbers and the situation would be very interesting to study in the future to see where the few-body character spreads out as function of system size. Another very noteworthy feature of our study is that among the excited states for balanced systems with equal numbers of bosons and fermions 'democratically' fermionized states appear. These states have densities identical to a system of identical fermions with the same total number of particles, but this is the case for both the fermionic and the bosonic density profile. This is, however, not the case for the five particle imbalanced systems that we have studied, and again indicates a pronounced odd-even effect in these systems.

Our focus here has been on repulsive interactions and we have not touched on the case with attractive interactions where one has additional (deeply) bound states 
in the spectrum. For the DMRG method this may be rather difficult to describe but should in principle be possible. For the analytical results at very large interaction strength there is an interesting mapping from the repulsive to the attractive side as recently pointed out by [78] in the context of effective spin chain models for the strongly interacting regime. The statement is that upon changing the sign of the interaction while simultaneously changing bosons for fermions will only change the spin chain Hamiltonian by an overall sign. This implies that the corresponding spectrum is inverted. The presence of this mapping in our results can be seen in several places. In the balanced $2+2$ system in figure 1 this is seen by comparing states 0 and 5, 1 and 4, as well as 2 and 3, all of which have identical densities if bosons and fermions are interchanged. The same is true for the $3+3$ case although we have not displayed the full spectrum here. For the imbalanced cases with five bodies, one should compare states 4 and 5 in figures 4 and 5 which are seen to be the same under exchange of fermions and bosons. In this way, we can already infer information about the strongly attractive regime from our data, although not for the deeper bound states that also arise with strong attraction.

\section{ACKNOWLEDGMENTS}

This work was supported by the Danish Council for Independent Research DFF Natural Sciences, the DFF Sapere Aude program, and the Villum Kann Rasmussen foundation. The authors thank M. E. S. Andersen, N. J. S. Loft, A. S. Jensen, D. V. Fedorov, M. Valiente and U. Schollwöck for discussions, and in particular we thank F. Deuretzbacher for extended discussions and for pointing out a missing factor of half in our bosonic interaction terms. Finally, we thank D. Pecak for discussion of results and comparison of our calculations to results obtained using exact diagonalization.

\section{REFERENCES}

[1] Elliott H Lieb and Daniel C Mattis. Mathematical physics in one dimension: exactly soluble models of interacting particles. Academic Press, 2013.

[2] M. Abramowitz and I. A. Stegun. Handbook of mathematical functions with formulas, graphs, and mathematical tables. 1965.

[3] R.J. Baxter. Exactly Solved Models in Statistical Mechanics. Elsevier Science, 2016. ISBN 9781483265940. URL https://books.google.dk/books?id=egtcDAAAQBAJ

[4] T. Giamarchi. Quantum Physics in One Dimension. International Series of Monographs on Physics. Clarendon Press, 2003. ISBN 9780198525004. URL https://books.google.dk/books?id=GVeuKZLGMZOC

[5] M. Plischke and B. Bergersen. Equilibrium Statistical Physics. World Scientific, 2006. ISBN 9789812560483. URL https://books.google.dk/books?id=KYu7igYEkhwC

[6] Immanuel Bloch, Jean Dalibard, and Wilhelm Zwerger. Many-body physics with ultracold gases. Rev. Mod. Phys., 80:885-964, Jul 2008. doi:10.1103/RevModPhys.80.885. URL http://link.aps.org/doi/10.1103/RevModPhys.80.885

[7] Maciej Lewenstein, Anna Sanpera, Veronica Ahufinger, Bogdan Damski, Aditi Sen(De), and Ujjwal Sen. Ultracold atomic gases in optical lattices: mimicking condensed matter physics and beyond. Advances in Physics, 56(2):243-379, 2007. doi:10.1080/00018730701223200. URL http://dx.doi .org/10.1080/00018730701223200.

[8] Tilman Esslinger. Fermi-hubbard physics with atoms in an optical lattice. Annual Review of Condensed Matter Physics, 1(1):129-152, 2010. doi. 10.1146/annurev-conmatphys-070909-104059. URL http://dx.doi.org/10.1146/annurev-conmatphys-070909-[11640

[9] Henning Moritz, Thilo Stöferle, Michael Köhl, and Tilman Esslinger. Exciting collective oscillations in a trapped $1 \mathrm{~d}$ gas. Phys. Rev. Lett., 91:250402, Dec 2003. doi:10.1103/PhysRevLett.91.250402 URL http://link.aps.org/doi/10.1103/PhysRevLett.91.250402

[10] Thilo Stöferle, Henning Moritz, Christian Schori, Michael Köhl, and Tilman Esslinger. Transition from a strongly interacting 1d superfluid to a mott insulator. Phys. Rev. Lett., 92:130403, Mar 2004. doi:10.1103/PhysRevLett.92.130403. URL http://link.aps.org/doi/10.1103/PhysRevLett.92.130403

[11] Toshiya Kinoshita, Trevor Wenger, and David S. Weiss. Observation of a one-dimensional tonksgirardeau gas. Science, 305(5687):1125-1128, 2004. ISSN 0036-8075. doi:10.1126/science.110070. URL http://science.sciencemag.org/content/305/5687/1125

[12] Toshiya Kinoshita, Trevor Wenger, and David S. Weiss. A quantum newton's cradle. Nature, 440(7086):900-903, Apr 2006. ISSN 0028-0836. doi:10.1038/nature04693. URL http://dx .doi .org/10.1038/nature04693

[13] Belen Paredes, Artur Widera, Valentin Murg, Olaf Mandel, Simon Folling, Ignacio Cirac, Gora V. Shlyapnikov, Theodor W. Hansch, and Immanuel Bloch. Tonks-girardeau gas of ultracold atoms in an optical lattice. Nature, 429(6989):277-281, May 2004. ISSN 0028-0836. doi:10.1038/nature02530. URL http://dx.doi.org/10.1038/nature02530.

[14] Elmar Haller, Mattias Gustavsson, Manfred J. Mark, Johann G. Danzl, Russell Hart, Guido Pupillo, and Hanns-Christoph Nägerl. Realization of an excited, strongly correlated quantum gas phase. Science, 325(5945):1224-1227, 2009. ISSN 0036-8075. doi:10.1126/science.1175850. URL http://science.sciencemag.org/content/325/5945/1224. 40159ar Haller, Russell Hart, Manfred J Mark, Johann G Danzl, Lukas Reichsöllner, Mattias Gustavsson, Marcello Dalmonte, Guido Pupillo, and Hanns-Christoph Nägerl. 
Pinning quantum phase transition for a luttinger liquid of strongly interacting bosons. Nature, 466(7306):597-600, 2010.

[16] Guido Pagano, Marco Mancini, Giacomo Cappellini, Pietro Lombardi, Florian Schafer, Hui Hu, XiaJi Liu, Jacopo Catani, Carlo Sias, Massimo Inguscio, and Leonardo Fallani. A one-dimensional liquid of fermions with tunable spin. Nat Phys, 10 (3):198-201, Mar 2014. ISSN 1745-2473. URL http://dx.doi.org/10.1038/nphys2878 Letter.

[17] Onofrio, Roberto. Cooling and thermometry of atomic Fermi gases Physics - Uspekhi, 59:11291153, 2016. doi:10.3367/UFNr.2016.07.037873. URL http://dx.doi.org/10.3367/UFNr.2016.07.037873.

[18] F. Serwane, G. Zürn, T. Lompe, T. B. Ottenstein, A. N. Wenz, and S. Jochim. Deterministic preparation of a tunable few-fermion system. Science, 332(6027):336-338, 2011. ISSN 0036-8075. doi:10.1126/science.1201351. URL http://science.sciencemag.org/content/332/6027/336

[19] G. Zürn, F. Serwane, T. Lompe, A. N. Wenz, M. G. Ries, J. E. Bohn, and S. Jochim. Fermionization of Two Distinguishable Fermions. Physical Review Letters, 108(7):075303, February 2012. doi: 10.1103/PhysRevLett.108.075303.

[20] G. Zürn, A. N. Wenz, S. Murmann, A. Bergschneider, T. Lompe, and S. Jochim. Pairing in few-fermion systems with attractive interactions. Phys. Rev. Lett. 111:175302, Oct 2013. doi:10.1103/PhysRevLett.111.175302. URL http://link.aps.org/doi/10.1103/PhysRevLett.111.175302

[21] A. N. Wenz, G. Zürn, S. Murmann, I. Brouzos, T. Lompe, and S. Jochim. From few to many: Observing the formation of a fermi sea one atom at a time. Science, 342(6157):457-460, 2013. ISSN 0036-8075. doi:10.1126/science.1240516. URL http://science.sciencemag.org/content/342/6157/457

[22] S. Murmann, F. Deuretzbacher, G. Zürn, J. Bjerlin, S. M. Reimann, L. Santos, T. Lompe, and S. Jochim. Antiferromagnetic heisenberg spin chain of a few cold atoms in a one-dimensional trap. Phys. Rev. Lett., 115:215301, Nov 2015. doi:10.1103/PhysRevLett.115.215301. URL http://link.aps.org/doi/10.1103/PhysRevLett.115.215361:

[23] Simon Murmann, Andrea Bergschneider, Vincent M. Klinkhamer, Gerhard Zürn, Thomas Lompe, and Selim Jochim. Two fermions in a double well: Exploring a fundamental building block of the hubbard model. Phys. Rev. Lett., 114:080402, Feb 2015. doi:10.1103/PhysRevLett.114.080402. URL http://link.aps.org/doi/10.1103/PhysRevLett.114.080402

[24] Zinner, Nikolaj Thomas. Exploring the few- to many-body crossover using cold atoms in one dimension. EPJ Web of Conferences, 113:01002, 2016. doi:10.1051/epjconf/201611301002 URL http://dx.doi.org/10.1051/epjconf/201611301002.

[25] Johannes Hofmann, Alejandro M. Lobos, and Victor Galitski. Parity effect in a mesoscopic fermi gas. Phys. Rev. A, 93:061602, Jun 2016. doi:10.1103/PhysRevA.93.061602. URL http://link.aps.org/doi/10.1103/PhysRevA.93.061602

[26] H. Bethe. Zur theorie der metalle. Zeitschrift für Physik, 71(3):205-226, $1931 . \quad$ ISSN 0044-3328. doi:10.1007/BF01341708. URL http://dx.doi.org/10.1007/BF01341708
[27] C. N. Yang. Some exact results for the manybody problem in one dimension with repulsive deltafunction interaction. Phys. Rev. Lett., 19:1312-1315, Dec 1967. doi:10.1103/PhysRevLett.19.1312 URL http://link.aps.org/doi/10.1103/PhysRevLett.19.1312.

[28] N Oelkers, M T Batchelor, M Bortz, and XW Guan. Bethe ansatz study of one-dimensional bose and fermi gases with periodic and hard wall boundary conditions. Journal of Physics A: Mathematical and General, 39(5):1073, 2006. URL http://stacks.iop.org/0305-4470/39/i=5/a=005

[29] Yajiang Hao, Yunbo Zhang, J. Q. Liang, and Shu Chen. Ground-state properties of one-dimensional ultracold bose gases in a hard-wall trap. Phys. Rev. A. 73:063617, Jun 2006. doi:10.1103/PhysRevA.73.063617. URL http://link.aps.org/doi/10.1103/PhysRevA.73.063617

[30] Lewi Tonks. The complete equation of state of one, two and three-dimensional gases of hard elastic spheres. Phys. Rev., 50:955-963, Nov $1936 . \quad$ doi:10.1103/PhysRev.50.955 URL http://link.aps.org/doi/10.1103/PhysRev.50.955.

[31] M. Girardeau. Relationship between systems of impenetrable bosons and fermions in one dimension. Journal of Mathematical Physics, 1(6):516-523, 1960. doi:http://dx.doi.org/10.1063/1.1703687. URL http://scitation.aip.org/content/aip/journal/jmp/1/6/10.106

[32] Xi-Wen Guan, Murray T. Batchelor, and Chaohong Lee. Fermi gases in one dimension: From bethe ansatz to experiments. Rev. Mod. Phys. 85:1633-1691, Nov 2013. doi:10.1103/RevModPhys.85.1633. URL http://link.aps.org/doi/10.1103/RevModPhys.85.1633

[33] F Anvari Vind, Angela Foerster, Ivan S Oliveira, Roberto Silva Sarthour, Diogo de Oliveira Soares-Pinto, Alexandre Martins de Souza, and Itzhak Roditi. Experimental realization of the yang-baxter equation via $\mathrm{nmr}$ interferometry. Scientific reports, 6, 2016.

[34] N. Spethmann, F. Kindermann, S. John, C. Weber, D. Meschede, and A. Widera. Inserting single cs atoms into an ultracold rb gas. Applied Physics B. 106(3):513-519.2012. 2. ISSN 1432-0649. doi:10.1007/s00340-011-4868-6. URL http://dx.doi.org/10.1007/s00340-011-4868-6

G. Zürn, A. N. Wenz, S. Murmann, A. Bergschneider, T. Lompe, and S. Jochim. Pairing in few-fermion systems with attractive interactions. Phys. Rev. Lett., 111:175302, Oct 2013. doi:10.1103/PhysRevLett.111.175302. URL http://link.aps.org/doi/10.1103/PhysRevLett.111.175302.

[36] Cheng Chin, Rudolf Grimm, Paul Julienne, and Eite Tiesinga. Feshbach resonances in ultracold gases. Rev. Mod. Phys., 82:1225-1286, April 2010. doi:10.1103/RevModPhys.82.1225. URL http://link.aps.org/doi/10.1103/RevModPhys.82.1225

[37] M. Olshanii. Atomic scattering in the presence of an external confinement and a gas of impenetrable bosons. Phys. Rev. Lett., 81:938-941, Aug 1998. doi:10.1103/PhysRevLett.81.938. URL http://link.aps.org/doi/10.1103/PhysRevLett.81.938

[38] Manuel Endres, Hannes Bernien, Alexander Keesling, Harry Levine, Eric R. Anschuetz, Alexandre Krajenbrink, Crystal Senko, Vladan Vuletic, Markus Greiner, and Mikhail D. Lukin. Atom-by-atom assembly of defect-free one-dimensional cold atom arrays. Science, 354(6315):1024-1027, 2016. ISSN 
0036-8075. doi:10.1126/science.aah3752 URL http://science.sciencemag.org/content/354/6315/1024

[39] Fabio Altomare and Albert M. Chang. OneDimensional Superconductivity in Nanowires. Wiley, 2013. ISBN 978-3-527-40995-2. URL http://eu.wiley.com/WileyCDA/WileyTitle/productCd-

[40] Tomasz Sowiński, Tobias Grass, Omjyoti Dutta, and Maciej Lewenstein. Few interacting fermions in a onedimensional harmonic trap. Phys. Rev. A, 88:033607, Sep 2013. doi:10.1103/PhysRevA.88.033607. URL http://link.aps.org/doi/10.1103/PhysRevA.88.033607

[41] F. Deuretzbacher, D. Becker, J. Bjerlin, S. M. Reimann, and L. Santos. Quantum magnetism without lattices in strongly interacting onedimensional spinor gases. Phys. Rev. A, 90:013611, Jul 2014. doi:10.1103/PhysRevA.90.013611. URL http://link.aps.org/doi/10.1103/PhysRevA.90.013611

[42] A. G. Volosniev, D. Petrosyan, M. Valiente, D. V. Fedorov, A. S. Jensen, and N. T. Zinner. Engineering the dynamics of effective spin-chain models for strongly interacting atomic gases. Phys. Rev. A, 91:023620, Feb 2015. doi:10.1103/PhysRevA.91.023620. URL http://link.aps.org/doi/10.1103/PhysRevA.91.023620

[43] Haiping $\mathrm{Hu}$, Liming Guan, and Shu Chen. Strongly interacting bose-fermi mixtures in one dimension. New Journal of Physics, 18(2):025009, 2016. URL http://stacks.iop.org/1367-2630/18/i=2/a=025009.

[44] Lijun Yang and Xiaoling Cui. Effective spin-chain model for strongly interacting one-dimensional atomic gases with an arbitrary spin. Phys. Rev. A, 93:013617, Jan 2016. doi:10.1103/PhysRevA.93.013617. URL http://link.aps.org/doi/10.1103/PhysRevA.93.013617

[45] Daniel Pecak, Mariusz Gajda, and Tomasz Sowiński. Two-flavour mixture of a few fermions of different mass in a one-dimensional harmonic trap. New Journal of Physics, 18(1):013030, 2016. URL http://stacks.iop.org/1367-2630/18/i=1/a=013030.

[46] Li Yang, Liming Guan, and Han Pu. Strongly interacting quantum gases in one-dimensional traps. Phys. Rev. A. 91:043634, Apr 2015. doi:10.1103/PhysRevA.91.043634. URL http://link.aps.org/doi/10.1103/PhysRevA.91.043634

[47] Steve Campbell, Miguel Ángel García-March, Thomás Fogarty, and Thomas Busch. Quenching small quantum gases: Genesis of the orthogonality catastrophe. Phys. Rev. A, 90:013617, Jul 2014. doi:10.1103/PhysRevA.90.013617. URL http://link.aps.org/doi/10.1103/PhysRevA.90.013617

[48] M A Garcia-March, B Julia-Diaz, G E Astrakharchik, Th Busch, J Boronat, and A Polls. Quantum correlations and spatial localization in one-dimensional ultracold bosonic mixtures. New Journal of Physics, 16(10):103004, 2014. URL http://stacks.iop.org/1367-2630/16/i=10/a=103004

[49] M. A. García-March, A. Yuste, B. JuliáDíaz, and A. Polls. Mesoscopic superpositions of tonks-girardeau states and the bosefermi mapping. Phys. Rev. A, 92:033621, Sep $2015 . \quad$ doi:10.1103/PhysRevA.92.033621. URL http://link.aps.org/doi/10.1103/PhysRevA.92.033621

[50] M A Garcis-March, A S Dehkharghani, and $\mathrm{N} \mathrm{T}$ Zinner. Entanglement of an impurity in a few-body one-dimensional ideal bose sys- tem. Journal of Physics B: Atomic, Molecular and Optical Physics, 49(7):075303, 2016. URL http://stacks.iop.org/0953-4075/49/i=7/a=075303.

[51] Sascha Zöllner, Hans-Dieter Meyer, and Peter Schmelcher. Composite fermion40 9 55 . ontml of dimensional bose-bose mixtures. Phys. Rev. A, 78:013629, Jul 2008. doi:10.1103/PhysRevA.78.013629. URL http://link.aps.org/doi/10.1103/PhysRevA.78.013629

[52] Emmerich Tempfli, Sascha Zöllner, and Peter Schmelcher. Binding between twocomponent bosons in one dimension. New Journal of Physics, 11(7):073015, 2009. URL http://stacks.iop.org/1367-2630/11/i=7/a=073015.

[53] Ioannis Brouzos and Peter Schmelcher. Construction of analytical many-body wave functions for correlated bosons in a harmonic trap. Phys. Rev. Lett., 108:045301, Jan 2012. doi:10.1103/PhysRevLett.108.045301. URL http://link.aps.org/doi/10.1103/PhysRevLett.108.045301.

[54] Ioannis Brouzos and Peter Schmelcher. Two-component few-fermion mixtures in a one-dimensional trap: Numerical versus analytical approach. Phys. Rev. A, 87:023605, Feb 2013. doi:10.1103/PhysRevA.87.023605. URL http://link.aps.org/doi/10.1103/PhysRevA.87.023605

[55] F. Deuretzbacher, K. Fredenhagen, D. Becker, K. Bongs, K. Sengstock, and D. Pfannkuche. Exact solution of strongly interacting quasi-one-dimensional spinor bose gases. Phys. Rev. Lett., 100:160405, Apr 2008. doi:10.1103/PhysRevLett.100.160405. URL http://link.aps.org/doi/10.1103/PhysRevLett.100.160405.

[56] Tomasz Sowiński, Mariusz Gajda, and Kazimierz Rzazewski. Pairing in a system of a few attractive fermions in a harmonic trap. EPL (Europhysics Letters), 109(2):26005, $2015 . \quad$ URL http://stacks.iop.org/0295-5075/109/i=2/a=26005.

[57] N T Zinner and A S Jensen. Comparing and contrasting nuclei and cold atomic gases. Journal of Physics G: Nuclear and Particle Physics, 40(5):053101, 2013. URL http://stacks.iop.org/0954-3899/40/i=5/a=053101.

[58] N J S Loft, L B Kristensen, A E Thomsen, and N T Zinner. Comparing models for the ground state energy of a trapped one-dimensional fermi gas with a single impurity. Journal of Physics B: Atomic, Molecular and Optical Physics, 49(12):125305, 2016. URL http://stacks.iop.org/0953-4075/49/i=12/a=125305

[59] N.J.S. Loft, L.B. Kristensen, A.E. Thomsen, A.G. Volosniev, and N.T. Zinner. Conan - the cruncher of local exchange coefficients for strongly interacting confined systems in one dimension. Computer Physics Communications, 209:171 - 182, 2016. ISSN 0010-4655. doi:http://dx.doi.org/10.1016/j.cpc.2016.08.021. URL http://www.sciencedirect.com/science/article/pii/S001046551

[60] Amin Dehkharghani, Artem Volosniev, Jonathan Lindgren, Jimmy Rotureau, Christian Forssén, Dmitri Fedorov, Aksel Jensen, and Nikolaj Zinner. Quantum magnetism in strongly interacting one-dimensional spinor bose systems. Scientific Reports, 5:10675 EP -, Jun 2015. URL http://dx.doi.org/10.1038/srep10675. Article.

[61] M. D. Girardeau and M. Olshanii. Theory of spinor fermi and bose gases in tight atom waveguides. Phys. Rev. A, 70:023608, Aug 2004. doi:10.1103/PhysRevA.70.023608. URL http://link.aps.org/doi/10.1103/PhysRevA.70.023608. 
[62] M. D. Girardeau and A. Minguzzi. Soluble models of strongly interacting ultracold gas mixtures in tight waveguides. Phys. Rev. Lett., 99:230402, Dec 2007. doi:10.1103/PhysRevLett.99.230402 URL http://link.aps.org/doi/10.1103/PhysRevLett.99.230402

[63] Jesper Levinsen, Pietro Massignan, Georg M. Bruun, and Meera M. Parish. Strong-coupling ansatz for the onedimensional fermi gas in a harmonic potential. Science Advances, 1(6), 2015. doi:10.1126/sciadv.1500197. URL http://advances.sciencemag.org/content/1/6/e1500197

[64] F. Deuretzbacher, D. Becker, and L. Santos. Momentum distributions and numerical methods for strongly interacting one-dimensional spinor gases. Phys. Rev. A. 94:023606, Aug 2016. doi:10.1103/PhysRevA.94.023606. URL http://link.aps.org/doi/10.1103/PhysRevA.94.023606

[65] Andrew P. Koller, Michael L. Wall, Josh Mundinger, and Ana Maria Rey. Dynamics of interacting fermions in spin-dependent potentials. Phys. Rev. Lett., 117:195302, Nov 2016. doi:10.1103/PhysRevLett.117.195302 URL http://link.aps.org/doi/10.1103/PhysRevLett.117.195302

[66] N. T. Zinner. Strongly interacting mesoscopic systems of anyons in one dimension. Phys. Rev. A, 92:063634, Dec 2015. doi:10.1103/PhysRevA.92.063634. URL http://link.aps.org/doi/10.1103/PhysRevA.92.063634

[67] Steven R. White. Density matrix formulation for quantum renormalization groups. Phys. Rev. Lett., 69:28632866, Nov 1992. doi:10.1103/PhysRevLett.69.2863. URL http://link.aps.org/doi/10.1103/PhysRevLett.69.2863

[68] Ulrich Schollwck. The density-matrix renormalization group in the age of matrix product states. Annals of Physics, 326(1):96 - 192, 2011. ISSN 0003-4916. doi:http://dx.doi.org/10.1016/j.aop.2010.09.012. URL http://www.sciencedirect.com/science/article/pii/so January 2011 Special Issue.

[69] M. Führinger, S. Rachel, R. Thomale, M. Greiter, and P. Schmitteckert. Dmrg studies of critical su(n) spin chains. Annalen der Physik, 17(12):922-936, 2008. ISSN 1521-3889. doi:10.1002/andp.200810326. URL http://dx.doi.org/10.1002/andp.200810326.

[70] M L Wall and Lincoln D Carr. Out-of-equilibrium dynamics with matrix product states. New Journal of Physics, 14(12):125015, 2012. URL http://stacks.iop.org/1367-2630/14/i=12/a=125015

[71] Bess Fang, Patrizia Vignolo, Mario Gattobigio, Christian Miniatura, and Anna Minguzzi. Exact solution for the degenerate ground-state manifold of a strongly interacting one-dimensional bosefermi mixture. Phys. Rev. A, 84:023626, Aug 2011. doi:10.1103/PhysRevA.84.023626. URL http://link.aps.org/doi/10.1103/PhysRevA.84.023626

[72] Haiping $\mathrm{Hu}$, Liming Guan, and Shu Chen. Strongly interacting bose-fermi mixtures in one dimension. New Journal of Physics, 18(2):025009, 2016. URL http://stacks.iop.org/1367-2630/18/i=2/a=025009.

[73] M. E. S. Andersen, A. S. Dehkharghani, A. G. Volosniev, E. J. Lindgren, and N. T. Zinner. An interpolatory ansatz for describing one-dimensional confined Fermi systems.
ArXiv e-prints, December 2015.

[74] A. G. Volosniev, D. V. Fedorov, A. S. Jensen, M. Valiente, and N. T. Zinner. Strongly interacting confined quantum systems in one dimension. Nature Communications, 5:5300, November 2014. doi:10.1038/ncomms6300.

[75] Artem G. Volosniev, Dmitri V. Fedorov, Aksel S. Jensen, Nikolaj T. Zinner, and Manuel Valiente. Multicomponent strongly interacting few-fermion systems in one dimension. Few-Body Systems, 55(8):839-842, 2014. ISSN 1432-5411. doi:10.1007/s00601-013-0776-0. URL http://dx.doi.org/10.1007/s00601-013-0776-0

[76] A S Dehkharghani, A G Volosniev, and N T Zinner. Impenetrable mass-imbalanced particles in one-dimensional harmonic traps. Journal of Physics B: Atomic, Molecular and Optical Physics, 49(8):085301, 2016. URL http://stacks.iop.org/0953-4075/49/i=8/a=085301.

[77] Filipe F. Bellotti, Amin S. Dehkharghani, and Nikolaj T. Zinner. Comparing numerical and analytical approaches to strongly interacting two-component mixtures in one dimensional traps. The European Physical Journal D, 71(2):37, 2017. ISSN 1434-6079. doi:10.1140/epjd/e2017-70650-8. URL http://dx.doi.org/10.1140/epjd/e2017-70650-8.

[78] F Deuretzbacher, D Becker, J Bjerlin, SM Reimann, and L Santos. Spin-chain model for strongly interacting one-dimensional bose-fermi mixtures. arXiv preprint arXiv:1611.04418, 2016.

[79] Kunal K. Das. Bose-fermi mixtures in one dimension. Phys. Rev. Lett. 90:170403, May 2003. doi:10.1103/PhysRevLett.90.170403. URL http://link.aps.org/doi/10.1103/PhysRevLett.90.170403

[80] M. A. Cazalilla and A. F. Ho. Instabilities in binary mixtures of one-dimensional quantum deS000349161000t1758.ses. Phys. Rev. Lett., 91:150403, Oct 2003. doi:10.1103/PhysRevLett.91.150403. URL http://link.aps.org/doi/10.1103/PhysRevLett.91.150403

[81] Adilet Imambekov and Eugene Demler. Exactly solvable case of a one-dimensional bose fermi mixture. Phys. Rev. A, 73:021602, Feb 2006. doi:10.1103/PhysRevA.73.021602. URL http://link.aps.org/doi/10.1103/PhysRevA.73.021602

[82] Hongmei Wang, Yajiang Hao, and Yunbo Zhang. Density-functional theory for onedimensional harmonically trapped bose-fermi mixture. Phys. Rev. A, 85:053630, May 2012. doi:10.1103/PhysRevA.85.053630. URL http://link.aps.org/doi/10.1103/PhysRevA.85.053630

[83] J. Decamp, P. Armagnat, B. Fang, M. Albert, A. Minguzzi, and P. Vignolo. Exact density profiles and symmetry classification for strongly interacting multicomponent Fermi gases in tight waveguides. ArXiv eprints, March 2016.

[84] M. L. Wall and L. D. Carr. Open Source TEBD. https://inside.mines.edu/ lcarr/software.html, 2009. URL https://inside.mines.edu/ lcarr/software.html

[85] iTensor project. http://itensor.org/index.html, 2016. URL https ://inside.mines . edu/ lcarr/software.html 